\title{
Profiles of brain magnetic activity during a memory task in patients with Alzheimer's disease and in non-demented elderly subjects, with or without depression
}

\author{
F Maestú, A Fernandez, P G Simos, M I López-Ibor, P Campo, J Criado, A Rodriguez-Palancas, \\ F Ferre, C Amo, T Ortiz
}

J Neurol Neurosurg Psychiatry 2004;75:1160-1162. doi: 10.1136/jnnp.2003.022376

The presence of depression is common among the elderly and it often complicates the early diagnosis of Alzheimer's disease (AD). In this study, we searched for brain activity measures that characterise AD. We compared brain magnetic activity profiles during a memory task, obtained from patients with $A D$, elderly patients with late onset depression, and age matched volunteers without history of neurological or psychiatric disease. AD patients showed significantly reduced activity in left temporal lobe regions during late portions of the event related magnetic response (400 ms or later after stimulus onset), compared with both groups of patients who did not present with serious cognitive decline. This finding highlights the potential usefulness of MEG protocols supporting the differential diagnosis of $A D$ and major depression related cognitive decline in the elderly.

$\mathrm{T}$ he association between late onset depression and other conditions that affect cognitive function in the elderly, such as Alzheimer's disease (AD) and mild cognitive impairment (MCI), is increasingly becoming the focus of attention in neurology and psychiatry since some studies have indicated that symptoms of depression may be present in early stages of $\mathrm{AD}^{1}$ and represent a predictive factor of subsequent dementia. ${ }^{2}$ The relationship between cognitive and neuropsychiatric symptoms is expected to have serious implications for diagnosis and treatment, and for understanding the underlying pathophysiology of $\mathrm{AD}$ and MCI. Recent magnetoencephalographic (MEG) studies $^{34}$ have reported time dependent differences in stimulus evoked activity between $\mathrm{AD}$ patients and healthy controls. In this study, we take advantage of the temporal resolution of MEG to extend previous findings by examining $\mathrm{AD}$ patients, elderly patients with major depression, and elderly control subjects.

\section{METHODS}

Twenty two elderly patients were assigned to two diagnostic groups: 12 patients fulfilling the NINCDS-ADRDA criteria of probable AD (mean (SD) age 63 (3) years) without symptoms of depression and 10 age matched patients (mean (SD) age 61 (5) years) diagnosed with major depression of late onset (mean (SD) duration of illness 3 (0.2) years). In addition, 10 elderly individuals with no history of neurological or psychiatric illness served as controls, matched on age with each of the patient groups (mean (SD) age 62 (4) years). All subjects received general medical, neurological, psychiatric, and psychological evaluations, and a brain MRI scan to rule out involvement of other types of brain damage (such as tumour or stroke). In addition, all participants were assessed using the Spanish version of the Mini Mental State Examination (MMSE) ${ }^{5}$ and the Hamilton depression scale. ${ }^{6}$ The only significant mean (SD) group differences on the MMSE were those between the AD patients (18.6 (2.3)) and the other two groups (depression patients: 32.3 (2.2); normal controls: 34 (1.2); $\mathrm{p}<0.00001)$. The group of patients with depression showed higher scores on the Hamilton depression scale (22.3 (3.3)), than both the control and AD groups (3.6 (1.3), $\mathrm{t}_{18}=-16.6, \mathrm{p}<0.001$; and 4 (1.5), $\mathrm{t}_{20}=17.15$, $\mathrm{p}<0.001$, respectively).

MEG recordings were obtained in the context of a modified version of Sternberg's letter probe task (for a detailed description see Maestu et $a l^{3}{ }^{4}$ ). Evoked magnetic activity was recorded with a whole head neuromagnetometer equipped with 148 axial magnetometer sensors (WH2500, 4-D Neuroimaging, San Diego, CA, USA). Examples of event related magnetic responses and isofield contour maps associated with acceptable dipolar activity sources in the left temporal lobe are presented in fig 1. Recording, analysis, and MEG-MRI co-registration procedures were identical to those used in our previous reports. ${ }^{3}{ }^{4}$ Based on the results of our previous studies on $\mathrm{AD}$ and on inspection of individual activation maps derived from the participants in the present study, statistical analyses were focused on activity sources located in the following brain regions: sensorimotor, insular, and ventrolateral frontal cortices (Brodmann's areas 1, 2, 3, $4,6,44,45)$, henceforth referred to as "cluster of complex motor processing areas", dorsolateral prefrontal regions (DLP, including Brodmann's areas 9, 46, and the posterior third of area 8), the whole temporal lobe (including superior and middle temporal gyrus and hippocampus related structures), and inferior parietal regions. Time dependent variations in activation profiles were examined by placing activity sources in each area into two latency windows, one extending from 150 to $400 \mathrm{~ms}$ and the second between 400 and $800 \mathrm{~ms}$ after stimulus onset. The rationale for time window selection was based on our earlier studies, ${ }^{3}{ }^{4}$ where group effects were restricted to early portions of the magnetic response (150$400 \mathrm{~ms}$ ) for certain regions and to late portions (400-800 ms) for other regions. The number of reliably localised activity sources in each of these areas in each hemisphere and time window (normalised to the total number of activity sources in the entire brain in each participant) served as the dependent measure in the statistical analyses.

\section{RESULTS}

As expected, $\mathrm{AD}$ patients performed worse than the other two groups on the experimental task. Mean per cent of correctly

Abbreviations: $A D$, Alzheimer's disease; MEG, magnetoencephalography; MCl, mild cognitive impairment; MMSE, Mini Mental State Examination 


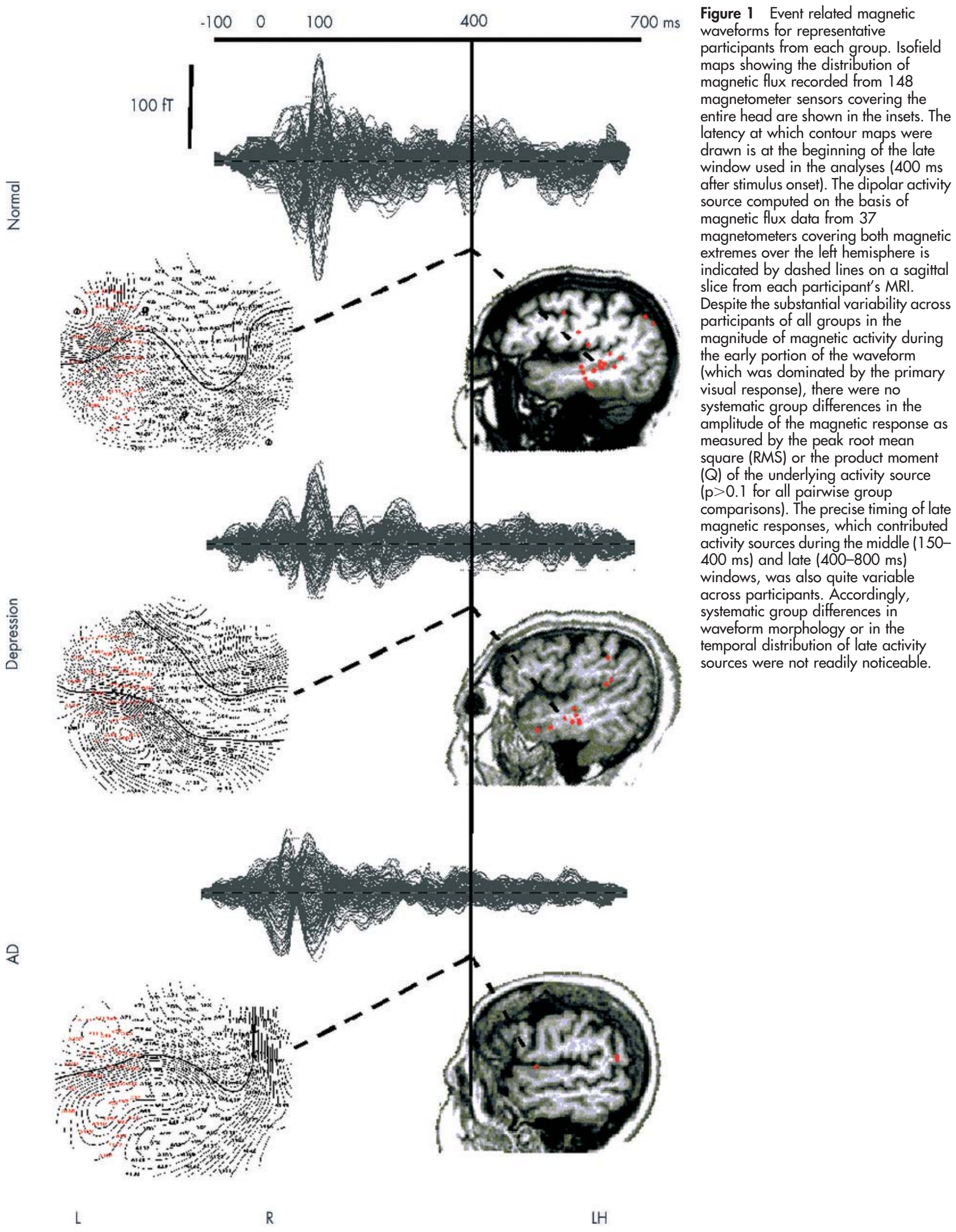

detected targets was $89.6 \%, 96.8 \%$ and $96 \%$ for the $\mathrm{AD}$ patients, depression patients, and normal controls, respectively. Corresponding false alarm rates were $44.4 \%, 0.56 \%$, and $0.3 \%$.

Normalised data were entered into a one way analysis of coveariance with area (4), hemisphere (2), and time window (2) as the repeated measures factors, group as an inter-subjects factor with three levels (elderly controls, depression patients, and AD patients), and age (in years) as an intra-groups covariate. The analysis revealed a four-way interaction $\left(\mathrm{F}_{(6,84)}=5.45, \mathrm{p}<0.003\right)$. Follow up three way analyses of covariance revealed a significant area of hemisphere by group interaction for the late window $\left(\mathrm{F}_{(6,84)}=\right.$ $6.81, \mathrm{p}<0.004)$. Further one way analysis of covariance tests 


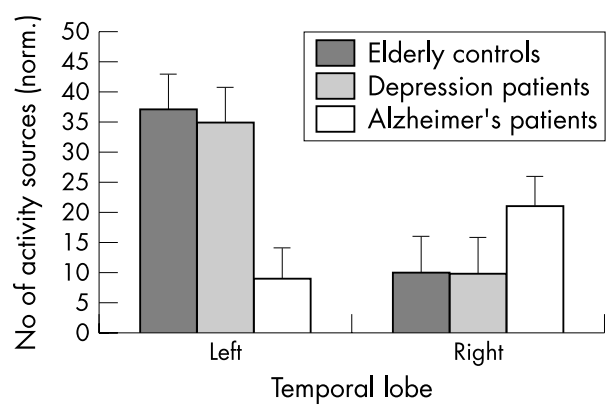

Figure 2 Average degree of activity in temporal lobe regions indicated by the number of consecutive activity sources that were reliably localised in this area, computed separately for each participant group. The dependent variable is expressed as the proportion of temporal lobe sources in the total number of activity sources found in the entire brain for a given participant, to control for individual differences in the overall degree of brain activity.

revealed a significant group main effect for the number of activity sources in the left temporal lobe $\left(F_{(2,29)}=7.26\right.$, $\mathrm{p}<0.003)$. This interaction is displayed graphically in fig 2 . Pairwise contrasts (with p values corrected according to the Bonferroni method) indicated that the AD group displayed reduced activity in the left temporal lobe during the late window compared with both elderly controls $(p<0.007)$, and patients with depression $(\mathrm{p}<0.012)$. The group main effect for the right hemisphere temporal lobe was marginally significant, $\mathrm{F}_{(2,29)}=4.38, \mathrm{p}<0.022$, suggesting that $\mathrm{AD}$ patients may have shown increased activity in this region compared with either elderly controls $(p>0.055)$, or nondemented patients with depression $(p>0.050)$. None of the differences between the control and depression groups approached significance $(\mathrm{p}>0.5)$.

Measures of temporal lobe activity were significant predictors of cognitive status as indexed by individual scores on the MMSE. Systematic variance in the degree of late window activity (400-800 ms) in the left and right temporal lobes accounted for $39 \%$ of the variance in individual MMSE scores $\left(\mathrm{R}^{2}=0.39, \mathrm{~F}_{(2,31)}=11.04, \mathrm{p}<0.0003\right)$. Partial correlations between the dependent variable and each of the two independent variables suggested that increased late activity in the left temporal lobe was associated with better performance on the MMSE $(r=0.55)$, whereas increased late activity in the right temporal lobe was associated with worse performance $(\mathrm{r}=-0.30)$.

\section{DISCUSSION}

In this study we present evidence that differences in regional activity between $\mathrm{AD}$ patients and elderly controls without cognitive deficits cannot be accounted for by the higher incidence of late onset depression in the former group. AD patients showed a smaller number of activity sources in the left temporal region than both groups of cognitively intact participants (with or without depression), confirming our previous findings. ${ }^{3}{ }^{4}$ The replication of this finding with a different sample of $\mathrm{AD}$ patients highlights the potential clinical usefulness of MEG as an adjunct tool for the diagnosis of $\mathrm{AD}$. This regional activation profile was selectively present in response to target stimuli and was apparent during late portions of the event related magnetic response-that is, $400 \mathrm{~ms}$ or later after stimulus onset. Task specific hypoactivation of the left temporal lobe is often accompanied by spontaneous, focal slow wave activity originating from the same region, ${ }^{7}$ and correlates strongly with the degree of hippocampal atrophy in $\mathrm{AD}$ patients. ${ }^{4}$

Failure to find differences between control subjects and elderly patients with depression in the degree of regional activity associated with our working memory task is in agreement with neuropsychological and structural imaging findings. Although patients with depression often have memory problems, ${ }^{8}$ this deficit appears to be more closely linked to emotional issues than to a generalised cognitive decline, and it is certainly not as severe as in patients with AD. ${ }^{9}$

No differences between groups were found in rolandic, premotor, and inferior frontal regions, although increased activity in these regions was noted for the $\mathrm{AD}$ group in our previous reports. ${ }^{34}$ We argued at that time that this phenomenon reflected a compensatory tendency for the rapid memory trace decay postulated in $\mathrm{AD}$, forcing patients to depend more heavily upon an articulatory rehearsal process. Differences among the two studies could be attributed to the effects of age on the regional activation profiles: patients in our previous studies were much older ( $>70$ years) than patients in the present study ( $<70$ years). It is possible that the normal, age related neuronal loss and associated memory decline is compounded by neurodegerative processes in older $\mathrm{AD}$ patients, increasing the need to engage compensatory strategies and alternative neuronal pathways to support memory function.

\section{Authors' affiliations}

\section{F Maestú, A Fernandez, P Campo, J Criado, C Amo, T Ortiz,}

Magnetoencephalography Center Dr Pérez Modrego, Universidad

Complutense de Madrid, Madrid, Spain

F Maestú, Departamento de Psicología Básica II (procesos cognitivos), Facultad de Psicología, Universidad Complutense de Madrid, Madrid, Spain

A Fernandez, M I López-lbor, T Ortiz, Departamento de Psiquiatría, Facultad de Medicina, Universidad Complutense de Madrid, Madrid, Spain

P G Simos, Department of Psychology, University of Crete, Greece A Rodriguez-Palancas, Servicio de Psiquiatría, Hospital Central de la Defensa Madrid, Madrid, Spain

F Ferre, Servicio de Psiquiatría Hospital Gregorio Marañón, Madrid, Spain

Competing interests: none declared

Correspondence to: Dr T Ortiz, Centro de Magnetoencefalografía Dr Pérez Modrego, Pabellón, 8 Facultad de Medicina, Universidad Complutense de Madrid 28040, Madrid, Spain; cmeg@rect.ucm.es

Received 2 July 2003

Revised 7 November 2003

Accepted 14 November 2003

\section{REFERENCES}

1 Lyketsos CG, Olin J. Depression in Alzheimer's disease: overview and treatment. Biol Psychiatry 2002;52:243-52.

2 Jorm AF. Is depression a risk factor for dementia or cognitive decline? A review. Gerontology 2000;46:219-27.

3 Maestu F, Fernandez A, Simos PG, et al. Spatio-temporal patterns of brain magnetic activity during a memory task in Alzheimer's disease. Neuroreport $2001 ; 12: 3917-22$.

4 Maestu F, Arrazola J, Fernandez A, et al. Do cognitive patterns of brain magnetic activity correlate with hippocampal atrophy in Alzheimer's disease? J Neurol Neurosurg Psychiatry 2003;74:208-12.

5 Lobo A, Ezquerra J, Gomez Burgada F, et al. (Cognocitive mini-test (a simple practical test to detect intellectual changes in medical patients). Actas Luso Esp Neurol Psiquiatr Cienc Afines 1979;7:189-202.

6 Hamilton M. A rating scale for depression. J Neurol Neurosurg Psychiatry 1960;23:56-62.

7 Fernandez A, Arrazola J, Maestu F, et al. Correlations of hippocampal atrophy and focal low-frequency magnetic activity in Alzheimer disease: volumetric MR imaging-magnetoencephalographic study. AJNR Am J Neuroradiol 2003;24:481-7.

8 Zakzanis KK, Leach L, Kaplan E. On the nature and pattern of neurocognitive function in major depressive disorder. Neuropsychiatry Neuropsychol Behav Neurol 1998;1 1:111-19.

9 Sabe L, Jason L, Juejati M, et al. Dissociation between declarative and procedural learning in dementia and depression. J Clin Exp Neuropsychol $1995 ; 17: 841-8$. 